\title{
Enlarging ion-transfer micropore channels of hierarchical carbon nanocages for ultrahigh energy and power densities
}

\author{
Jin Zhao ${ }^{1,2}$, Hao Fan ${ }^{1}$, Guochang $\mathrm{Li}^{1}$, Qiang Wu${ }^{1}$, Lijun Yang ${ }^{1}$, Yanwen $\mathrm{Ma}^{2}$, Xizhang Wang ${ }^{{ }^{*}}$ and \\ Zheng $\mathrm{Hu}^{1 *}$
}

\begin{abstract}
Increasing the energy density of supercapacitor without sacrificing its high power is an everlasting pursuit in energy storage. Using ionic liquid electrolyte with high operating voltage can increase the energy density but usually at the expense of power density due to the large ion size, low ionic conductivity and high viscosity. Herein we demonstrate a simultaneous increase of the energy and power densities with ionic liquid electrolyte $\left(\mathrm{EMIMBF}_{4}\right)$ mainly by enlarging the ion-transfer micropore channels of the electrode material, i.e., the unique hierarchical carbon nanocages (hCNC). Boudouard reaction is adopted to tune the micropore size while remaining the hierarchical framework of hCNC. Meanwhile, the specific surface area, pore volume and conductivity are also increased under optimal activation temperature. Such a unique modification boosts the large-sized ion transfer, leading to the obvious decrease of equivalent series resistance and the dramatic increase of supercapacitive performance thereof. The optimized product exhibits an energy density up to $153.8 \mathrm{~W} \mathrm{~h} \mathrm{~kg}^{-1}$ at the power density of $1.8 \mathrm{~kW} \mathrm{~kg}^{-1}$, and maintains $54.0 \mathrm{~W} \mathrm{~h} \mathrm{~kg}^{-1}$ even at an ultrahigh power density of $480.1 \mathrm{~kW} \mathrm{~kg}^{-1}$. This study demonstrates an effective way to explore advanced electrode materials by the fine regulation of micropores and related properties.
\end{abstract}

Keywords: supercapacitors, ultrahigh energy and power densities, hierarchical carbon nanocages, micropore enlarging, ionic liquid electrolyte

\section{INTRODUCTION}

Advanced energy storage with both high energy and power densities is of vital importance to address the en- ergy demands and promote the development of portable electronics and electric vehicles. Supercapacitors are known far superior to batteries in terms of power density and cycling stability, but inferior in energy density. Hence the application of supercapacitors is usually restricted in the fields only needing high power. To broaden the applications, increasing the energy density without sacrificing the power density becomes a challenging topic [1-5]. The energy and power densities of a supercapacitor are given by the formulas of $E=C V^{2} / 2$ and $P=V^{2} / 4 R_{\mathrm{ESR}}$, respectively. Accordingly, exploring the high-capacitance (C) electrode materials with small equivalent series resistance $\left(R_{\mathrm{ESR}}\right)$ and expanding the device operating voltage $(V)$ are crucial to acquire both high $E$ and $P$. As known, ionic liquid electrolyte has high operating voltage up to $4 \mathrm{~V}$, and hence is usually used to enhance the energy density of supercapacitors. However, the use of ionic liquid electrolyte will lead to large $R_{\mathrm{ESR}}$ due to the large ion size, low ionic conductivity and high viscosity, thus resulting in a lower power density in comparison with the case in aqueous electrolyte [6-10]. The pore structure of the electrode material strongly influences the rate capability and the power density of a supercapacitor [11-16]. Consequently, optimizing the pore structure should be an effective way to compensate the power deterioration in the case of ionic liquid electrolyte.

In the past decade, abundant carbon-based materials in diverse forms and dimensions have been explored as supercapacitor electrode materials [17-22]. Our group reported the novel 3D hierarchical carbon nanocages (hCNC) featuring large specific surface area (SSA), co-

\footnotetext{
${ }^{1}$ Key Laboratory of Mesoscopic Chemistry of MOE and Jiangsu Provincial Lab for Nanotechnology, School of Chemistry and Chemical Engineering, Nanjing University, Nanjing 210023, China

${ }^{2}$ Jiangsu Key Lab for Organic Electronics and Information Displays \& Institute of Advanced Materials, Nanjing University of Posts and Telecommunications, Nanjing 210023, China

* Corresponding authors (emails: zhenghu@nju.edu.cn (Hu Z); wangxzh@nju.edu.cn (Wang X))
} 
existing micro-meso-macropore structure, high conductivity and tunable wettability. Specifically, the nanocages interconnect to form the micrometer-sized nanosheets, which further assemble into three-dimensional (3D) sphere-like particles. The micropores penetrating across the shell have the small pore sizes of ca. $0.6 \mathrm{~nm}[4,23,24]$. Such unique features result in the high supercapacitive performance, but still with the energy densities far below the batteries' [25-27]. We noticed that hCNC has the good wettability to ionic liquid of 1-ethyl3-methylimidazolium tetrafluoroborate $\left(\mathrm{EMIMBF}_{4}\right)$ with the operating voltage up to $4 \mathrm{~V}$, which is favorable for high energy density (Fig. S1) [27,28]. However the micropore channels of ca. $0.6 \mathrm{~nm}$ of hCNC is relatively small for the ion transfer of $\mathrm{EMIMBF}_{4}$ with the $\mathrm{EMIM}^{+}$ion size of $4.3 \AA \times 7.6 \AA$. In this study, Boudouard reaction, i.e., C $+\mathrm{CO}_{2}=2 \mathrm{CO}$, was applied to enlarge the micropore size by activating (etching) the hCNC material. With the optimal activation temperature, all the important parameters for supercapacitors such as the pore size distribution, pore volume, SSA and electron conductivity of hCNC are improved, leading to the much enhanced energy storage capability in $\mathrm{EMIMBF}_{4}$. The supercapacitor based on the activated hCNC (a-hCNC) at $900^{\circ} \mathrm{C}$ exhibits an ultrahigh energy density of $153.8 \mathrm{~W} \mathrm{~h} \mathrm{~kg}^{-1}$ at the power density of $1.8 \mathrm{~kW} \mathrm{~kg}^{-1}$, and maintains $54.0 \mathrm{~W} \mathrm{~h} \mathrm{~kg}^{-1}$ even under an ultrahigh power density of $480.1 \mathrm{~kW} \mathrm{~kg}^{-1}$, which locates at the top-level for carbon-based electrode materials.

\section{EXPERIMENTAL SECTION}

\section{Preparation of samples}

hCNC was prepared at $800^{\circ} \mathrm{C}$ by in situ $\mathrm{MgO}$ template method as described in our previous study [26]. In a typical procedure, basic magnesium carbonate $\left(4 \mathrm{MgCO}_{3} \cdot \mathrm{Mg}(\mathrm{OH})_{2} \cdot 5 \mathrm{H}_{2} \mathrm{O}\right)(4 \mathrm{~g})$ with a $3 \mathrm{D}$ hierarchical structure was placed into a quartz tube in a vertical furnace. For the growth of hCNC, the furnace was heated up to $800^{\circ} \mathrm{C}$ in $\mathrm{Ar}$ at the flow rate of $100 \mathrm{sccm}$ and heating rate of $10^{\circ} \mathrm{C} \mathrm{min}{ }^{-1}$. Then benzene was introduced into the tube at the feeding rate of $65 \mu \mathrm{L} \mathrm{min}{ }^{-1}$ by a syringe pump for $12 \mathrm{~min}$. During the growth, the sample was remixed by a flash of high-rate Ar flow of $1200 \mathrm{sccm}$ with the interval of $1 \mathrm{~min}$. The reactor was thus naturally cooled down to room temperature. The as-prepared sample was first stirred in $6 \mathrm{~mol} \mathrm{~L}^{-1} \mathrm{HCl}$ aqueous solution for $48 \mathrm{~h}$ to remove the $\mathrm{MgO}$ template and repeatedly washed with deionized water. Finally, the hCNC was obtained after drying at $80^{\circ} \mathrm{C}$ for $24 \mathrm{~h}$. For a-hCNC preparation, $50 \mathrm{mg}$ of obtained hCNC was placed into a quartz tube in a horizontal furnace. The furnace was first flushed with $\mathrm{Ar}$ for $20 \mathrm{~min}$ and then heated to the designated activation temperatures of 800,900 or $1000^{\circ} \mathrm{C}$, respectively, at a rate of $10^{\circ} \mathrm{C} \mathrm{min}{ }^{-1}$ and maintained for 1 or $2 \mathrm{~h}$ in 50 vol.\% $\mathrm{CO}_{2} / \mathrm{Ar}$ gaseous mixture at the flow rate of $100 \mathrm{sccm}$. After activation, the reactor was spontaneously cooled down to ambient temperature to obtain the products.

\section{Structural characterizations}

The morphologies and structures of the samples were characterized by scanning electron microscopy (SEM, Hitachi $\mathrm{S} 4800$ at $10 \mathrm{kV}$ ), transmission electron microscopy (TEM, JOEL JEM-2100F operated at $200 \mathrm{kV}$ ), Raman spectra (Witec alpha $300 \mathrm{SR}$ with an excitation laser of $488 \mathrm{~nm}$ ), X-ray photoelectron spectroscopy (XPS, Kratos AXIS Supra X-ray photoelectron spectrometer) and Fourier transform infrared spectroscopy (FTIR, Perkin-Elmer 100 FTIR spectrometer). $\mathrm{N}_{2}$ adsorption/ desorption isotherms were measured on Thermo Fisher Scientific Surfer Gas Adsorption Porosimeter at $77 \mathrm{~K}$. Before measurement, the sample was degassed at $300^{\circ} \mathrm{C}$ for $6 \mathrm{~h}$. The SSA was calculated using the BET (BrunauerEmmett-Teller) method based on the adsorption data in the linear relative pressure $\left(p / p_{0}\right)$ range of $0.05-0.3$. The pore size distribution was calculated from the corresponding adsorption branch of $\mathrm{N}_{2}$ isotherm by HorvathKawazoe method for micropore and Barrett-Joyner-Halenda method for meso- and macro-pore. The conductivity was measured by a four-wire method using a source measure unit (Keithley 6430) [26,29].

\section{Dynamic contact angle measurements}

The sample was first wetted with ethanol and then rolled into sheet on aluminum foil. After drying at $80^{\circ} \mathrm{C}$ for $12 \mathrm{~h}$, the evolution of the contact angle was recorded by the contact angle measurement system (OCA 30, DataPhysics Instruments $\mathrm{GmbH}$ ).

\section{Fabrication of supercapacitors}

The symmetric supercapacitor of coin-cell type was fabricated to evaluate the supercapacitive performance of the products. Briefly, $95 \mathrm{wt} \%$ product and $5 \mathrm{wt} \%$ polytetrafluoroethylene (PTFE, $60 \mathrm{wt} \%$ dispersion in water) were homogeneously mixed into a paste using an agate mortar and pestle. Then the mixture was rolled onto the stainless steel wire meshes $(316 \mathrm{~L}, 400$ meshes) with a diameter of $1.4 \mathrm{~cm}$. The electrodes/collectors were dried at $180^{\circ} \mathrm{C}$ for $6 \mathrm{~h}$ under high vacuum to completely remove the water. The dried electrodes/collectors were vacuum transferred into a glove box filled with Ar to construct symmetrical 
supercapacitors (CR2032) with the porous cellulose membrane as separator. The active material on each electrode was $1.0 \mathrm{mg}$, with the mass loading of $0.65 \mathrm{mg} \mathrm{cm}^{-2}$. The mass was measured by Sartorius Ultramicrobalance $(0.1 \mu \mathrm{g}$ resolution).

\section{Electrochemical characterizations and calculations}

All the supercapacitive performances were performed with a VMP3 Electrochemical Workstation (Bio-logic). The electrochemical impedance spectroscopy (EIS) was performed at open circuit potential with a sinusoidal signal over a frequency range from $100 \mathrm{kHz}$ to $10 \mathrm{mHz}$ at an amplitude of $10 \mathrm{mV}$. The cycling test was evaluated by galvanostatic charge-discharge (GCD) measurement.

Specific capacitance of the electrode material $\left(C_{\mathrm{s}}, \mathrm{F} \mathrm{g}^{-1}\right)$ was calculated from the chronopotentiometry $(\mathrm{CP})$ discharge curve:

$C_{\mathrm{s}}=\frac{2 \times I_{\text {cons }} \times \Delta t}{m \times \Delta V}$,

where $I_{\text {cons }}$ is the constant current in discharging, $m$ is the mass of active material on one electrode, $\Delta t$ is the discharge time, and $\Delta V$ is the voltage change during discharge (excluding the limited voltage (IR) drop).

Energy density $\left(E, \mathrm{~W} \mathrm{~h} \mathrm{~kg}^{-1}\right)$ of the cell was calculated by:

$E=\frac{C_{\mathrm{s}} \times \Delta V^{2}}{2 \times 4 \times 3.6}$

Average-power density $\left(P, \mathrm{~W} \mathrm{~kg}^{-1}\right)$ of the cell was obtained by:

$P=\frac{E \times 3600}{\Delta t}$

Maximum-power density $\left(P_{\max }, \mathrm{W} \mathrm{kg}^{-1}\right)$ of the cell was obtained by:

$P_{\max }=\frac{\Delta V^{2}}{8 \times R_{\mathrm{ESR}} \times m}$,

$R_{\mathrm{ESR}}=\frac{V_{\text {drop }}}{2 \times I_{\text {cons }}}$,

where $V_{\text {drop }}$ is the $I R$ drop between the first two points in the discharge curve, $R_{\mathrm{ESR}}$ is the equivalent series resistance.

\section{RESULTS AND DISCUSSION}

The a-hCNCs treated at 800,900 , and $1000^{\circ} \mathrm{C}$ for 1 or $2 \mathrm{~h}$ were designated as a-hCNC800-1h, a-hCNC900-1h, ahCNC1000-1h and a-hCNC1000-2h, respectively. Fig. 1 shows the change of pore size distribution of hCNC before and after activation. The $\mathrm{N}_{2}$ sorption isotherms for all the products are similar with each other, indicating the slight change in the pore structure. The steep uptake at
$P / P_{0}<0.01$ indicates the existence of abundant micropores, while the hysteresis loop at $0.42<P / P_{0}<1$ with no limiting adsorption at high $P / P_{0}$ corresponds to mesoand macropores (Fig. 1a) [30,31]. Both the SSA and the pore volume increase from $1890 \mathrm{~m}^{2} \mathrm{~g}^{-1}$ and $4.66 \mathrm{~cm}^{3} \mathrm{~g}^{-1}$ for hCNC to $2119 \mathrm{~m}^{2} \mathrm{~g}^{-1}$ and $4.75 \mathrm{~cm}^{3} \mathrm{~g}^{-1}$ for ahCNC800-1h, $2399 \mathrm{~m}^{2} \mathrm{~g}^{-1}$ and $5.26 \mathrm{~cm}^{3} \mathrm{~g}^{-1}$ for ahCNC900-1h, and $2776 \mathrm{~m}^{2} \mathrm{~g}^{-1}$ and $6.52 \mathrm{~cm}^{3} \mathrm{~g}^{-1}$ for ahCNC1000-1h, then slightly decrease to $2497 \mathrm{~m}^{2} \mathrm{~g}^{-1}$ and $5.03 \mathrm{~cm}^{3} \mathrm{~g}^{-1}$ for a-hCNC1000-2h (Table S1). Meanwhile, the micropore size gradually increases as reflected in the corresponding positive extending of the micropore size distribution curves (inset in Fig. 1b), which is indeed what we expected as schemed in Fig. 1c $[32,33]$. This result also indicates that the etching predominantly occurs on the micropore edge, where the carbon atoms are more active than those in the basal plane [34], as supported by our theoretical calculations (Fig. S2). The wellmaintained nanocage structure coupled with the enlarged micropore size should be responsible for the general increase of SSA and pore volume for the treated samples except for the over-treated a-hCNC1000-2h with somewhat collapsion of the nanocage structure [27]. SEM and TEM observations also confirm the similar structure and morphology of the 1h-treated samples to hCNC, as shown in Fig. 2 (Fig. S3). The enlarged micropore size is favorable for the ion transfer through the cages as schemed in Fig. 1d. Actually, we calculated the EMIM ${ }^{+}$ adsorption energy in the micropores with different sizes of $4 \AA \times 4 \AA, 6 \AA \times 6 \AA, 6 \AA \times 10 \AA, 10 \AA \times 10 \AA, 14 \AA \times$ $14 \AA$ and infinite (the edge model) by the using the density functional theory method. With increasing micropore size, the carbon and hydrogen numbers that can interact with $\mathrm{EMIM}^{+}$through van der Waals force decrease gradually, and converge to the case of edge. Therefore, pore widening can effectively promote the ion diffusion through the micropores (Fig. 1e).

After activation, the conductivity firstly increased from $663 \mathrm{~S} \mathrm{~m}^{-1}$ for hCNC to $734 \mathrm{~S} \mathrm{~m}^{-1}$ for a-hCNC800-1h and $794 \mathrm{~S} \mathrm{~m}^{-1}$ for a-hCNC900-1h, and then decreased to $573 \mathrm{~S} \mathrm{~m}^{-1}$ for a-hCNC1000-1h and $212 \mathrm{~S} \mathrm{~m}^{-1}$ for ahCNC1000-2h (Fig. S3d). Such a change could be attributed to the initial removal of the poorly crystalized carbon by $\mathrm{CO}_{2}$ etching followed by the regeneration of new defects of the destroyed conjugated structure of $\mathrm{sp}^{2}$ carbon due to the excessive activation with increasing activation temperature and time (Fig. S2) [35-38]. The increased micropore size, SSA and pore volume, as well as the well maintained or even enhanced conductivity after activation are expected to elevate the supercapacitive 


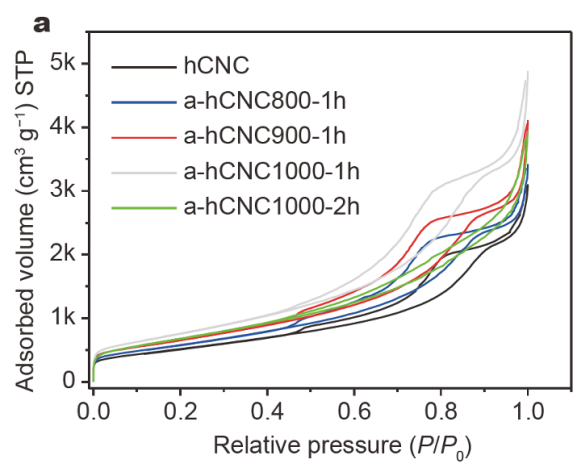

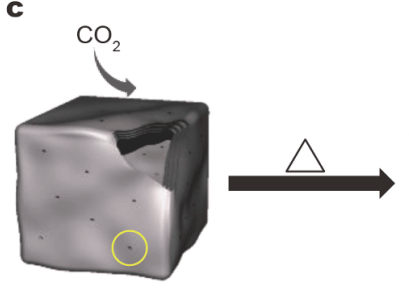

hCNC

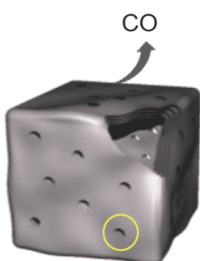

a-hCNC

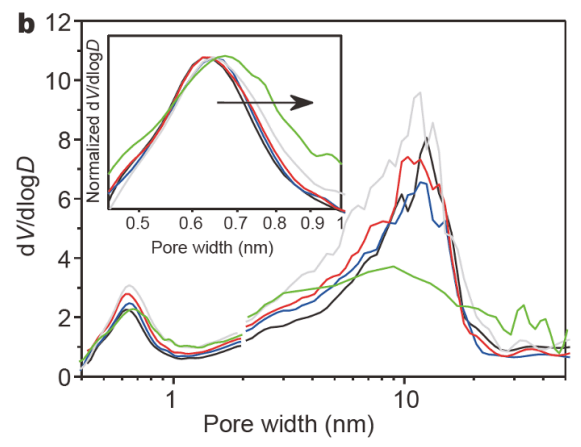

d

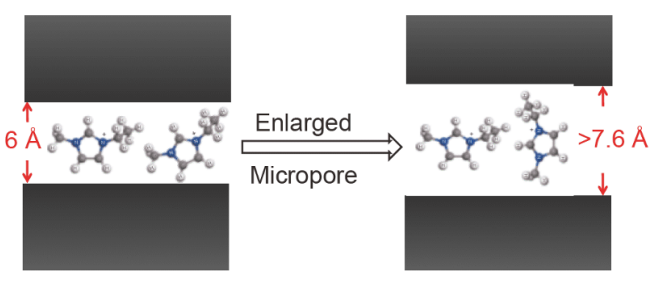

$\mathrm{hCNC}$

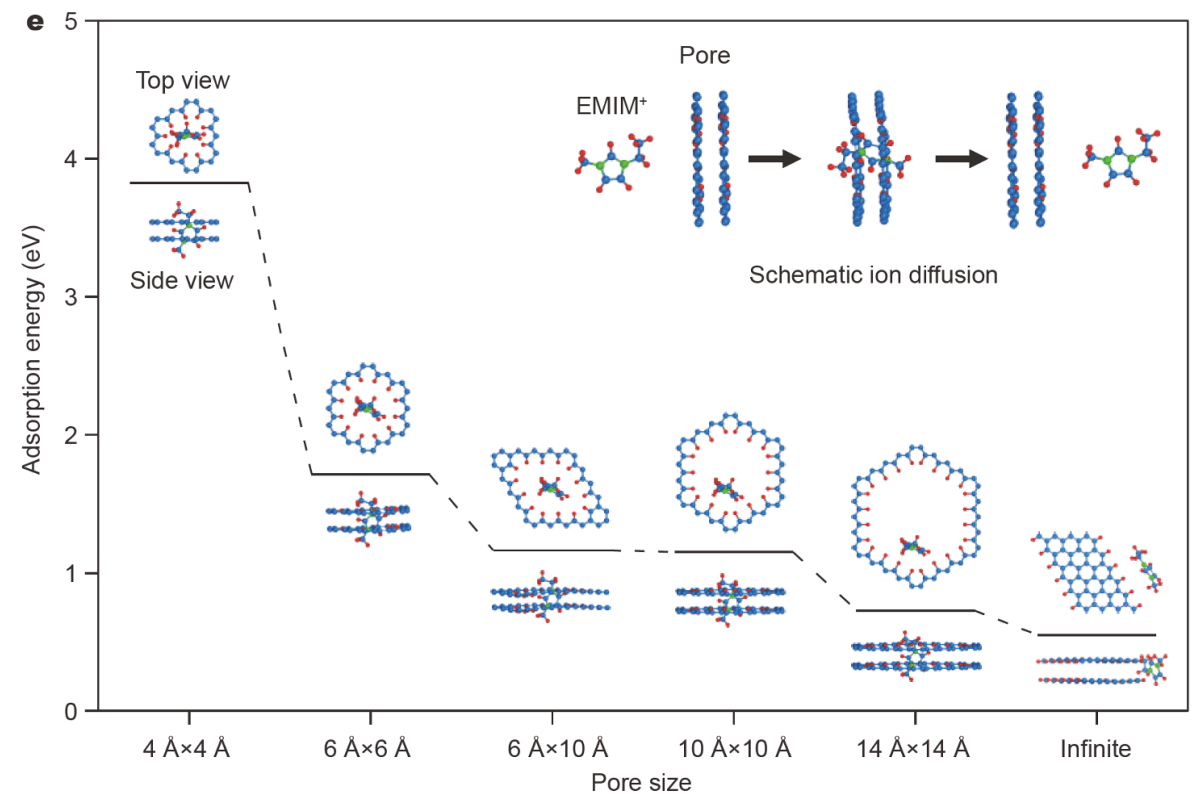

Figure 1 Pore structure evolution of hCNC before and after activation at 800 , 900, and $1000^{\circ} \mathrm{C}$. (a) $\mathrm{N}_{2}$ sorption isotherms. (b) Pore size distributions. Inset is the local enlargement of the micropore distributions. (c) Schematic of micropore size enlargement by Boudouard reaction. (d) Schematic EMIM $^{+}$transfer across the micropore channels of hCNC and a-hCNC with the latter case the easier due to the enlarged size. (e) Adsorption energy of $\mathrm{EMIM}^{+}$diffusion through the micropores with different sizes. Inset is the schematic ion diffusion through the micropore.

performances of hCNC in $\mathrm{EMIMBF}_{4}$.

The supercapacitive performances were evaluated by the two-electrode coin-type supercapacitors. Fig. 3 shows the electrochemical performances for the hCNC-, ahCNC800-1h-, a-hCNC900-1h- and a-hCNC1000-1hbased supercapacitors, denoted as hCNC-SC, a-
hCNC800-1h-SC, a-hCNC900-1h-SC and a-hCNC1000$1 \mathrm{~h}-\mathrm{SC}$, respectively. The typical cyclic voltammetry (CV) curves for the hCNC-SC and a-hCNC900-1h-SC show good rectangle-like shapes at the scan rate of $200 \mathrm{mV} \mathrm{s}^{-1}$, indicating the ideal capacitive behavior. With increasing scan rate up to $1000 \mathrm{mV} \mathrm{s}^{-1}$, the $\mathrm{CV}$ curves for the a- 


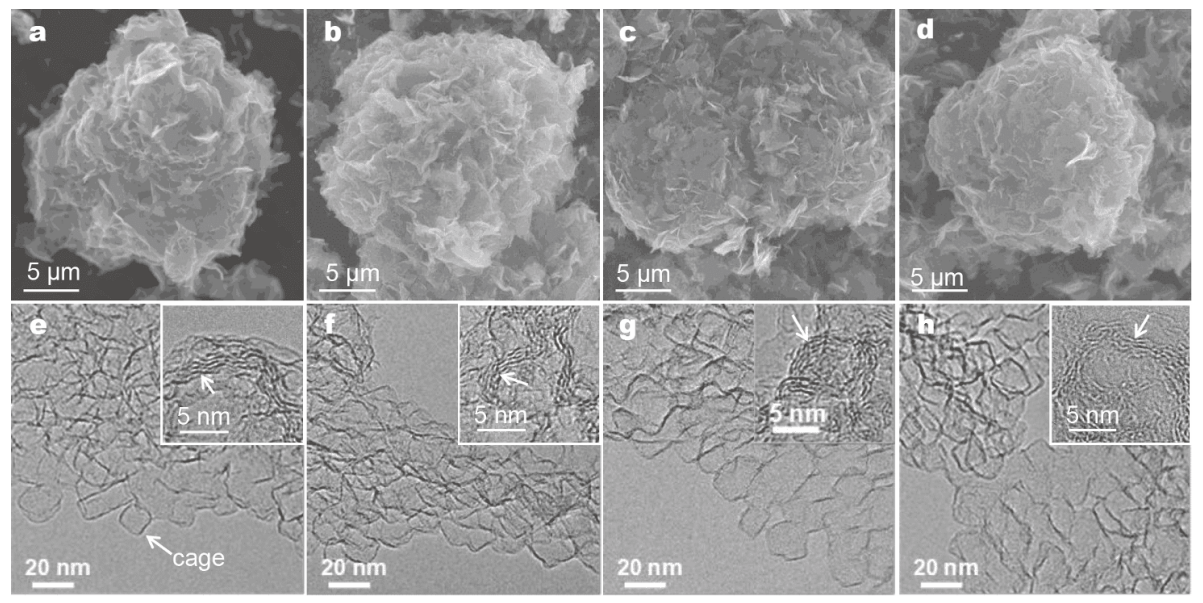

Figure 2 Morphological and structural characterizations of the products. (a-d) SEM images. (e-h) TEM images. (a, e) hCNC, (b, f) a-hCNC800-1h, (c, g) a-hCNC900-1h, (d, h) a-hCNC1000-1h. Insets in (e-h) are the corresponding high-resolution TEM images. Arrows indicate the broken fringes.

hCNC900-1h-SC is more close to rectangle than that for the hCNC-SC, demonstrating the better capacitive behavior with higher rate capability and smaller $R_{\mathrm{ESR}}$ (Fig. 3a) [25-28]. The weak humps in the CV curves of $200 \mathrm{mV} \mathrm{s}^{-1}$ indicate the slight contribution of pseudocapacitance, which should be caused by the small amount of oxygen-containing groups in the products $[26,39]$. The ahCNC800-1h-SC and a-hCNC1000-1h-SC also exhibit better capacitive behavior than the hCNC-SC, but slightly inferior to the a-hCNC900-1h-SC (Fig. S4). GCD curves show high symmetry and nearly linear slopes at the current density of $1 \mathrm{~A} \mathrm{~g}^{-1}$ for all the supercapacitors, but the discharge times for the a-hCNC-based supercapacitors are longer than that for the hCNC-SC, in agreement with the CV characterization results (Fig. 3a, b). At each current density, the $I R$ drops for all the ahCNC-based supercapacitors are smaller than that for the hCNC-SC, which also indicates the enhanced performance for the a-hCNC, with a-hCNC900-1h the best (Fig. $3 \mathrm{~b}$ and Figs S5, S6). At the current density of $1 \mathrm{~A} \mathrm{~g}^{-1}$, the specific capacitance obtained from the discharge curve is $236,265,278$ and $270 \mathrm{Fg}^{-1}$ for hCNC, ahCNC800-1h, a-hCNC900-1h and a-hCNC1000-1h, respectively, locating at the top-level for carbon-based materials, especially in ionic liquid electrolyte (Table S2) $[28,40-43]$. With increasing current density up to $200 \mathrm{~A} \mathrm{~g}^{-1}$, a-hCNC900-1h still maintains ca. $62.2 \%$ of its initial capacitance $\left(173 \mathrm{~F} \mathrm{~g}^{-1}\right)$, superior to the corresponding $37.4 \%, 53.2 \%$ and $52.6 \%$ for hCNC, ahCNC800-1h and a-hCNC1000-1h, respectively (Fig. 3c). The inferior supercapacitive performance for ahCNC1000-1h to a-hCNC900-1h could be attributed to the decreased conductivity for the former $[28,44,45]$.
Ragone plots further demonstrate the best performance of the a-hCNC900-1h-SC among the four supercapacitors, which delivers an ultrahigh energy density of $153.8 \mathrm{~W} \mathrm{~h} \mathrm{~kg}^{-1}$ at the average-power density of $1.8 \mathrm{~kW} \mathrm{~kg}^{-1}$ based on the weight of a-hCNC900-1h. Even at an ultrahigh power density of $480.1 \mathrm{~kW} \mathrm{~kg}^{-1}$, the energy density still reaches $54.0 \mathrm{~W} \mathrm{~h} \mathrm{~kg}^{-1}$, indicating the simultaneous high energy density and high power output (Fig. 3d). Actually, the energy storage capabilities of all the four samples locate at the top-level for carbon-based materials (Fig. 3d, e and Table S2) [28,40-43,46-50]. Such a high energy density is attributed to the large specific capacitance of a-hCNC900-1h and the high operating voltage up to $4 \mathrm{~V}$ in $\mathrm{EMIMBF}_{4}$. In addition, the ahCNC900-1h-SC displays an excellent stability, showing ca. $93.8 \%$ capacitance retention after 20,000 cycles at the high current density of $100 \mathrm{~A} \mathrm{~g}^{-1}$, with the Coulombic efficiency of ca.100\% through the testing cycles. The slight increase of capacitance at the beginning of cycling test could be attributed to the electrochemical activation (Fig. S7) [43]. Moreover, the cage structure of ahCNC900-1h is well maintained after cycling for 20,000 cycles (Fig. S8). These results indicate the high stability of the electrode material. Its theoretical maximum-power density, which is obtained to be $634 \mathrm{~kW} \mathrm{~kg}^{-1}$ at the current density of $20 \mathrm{~A} \mathrm{~g}^{-1}$, also locates at the top-level for carbon-based supercapacitors in nonaqueous electrolytes (Fig. S9 and Table S2). With increasing mass loading to higher values of 3.25 and $5.19 \mathrm{mg} \mathrm{cm}^{-2}$, the performance for a-hCNC900-1h decreases accordingly due to the increased ion transfer resistance, but still maintains at the high level (Fig. S10). The influences of defects and functional groups on the capacitive performance are very 

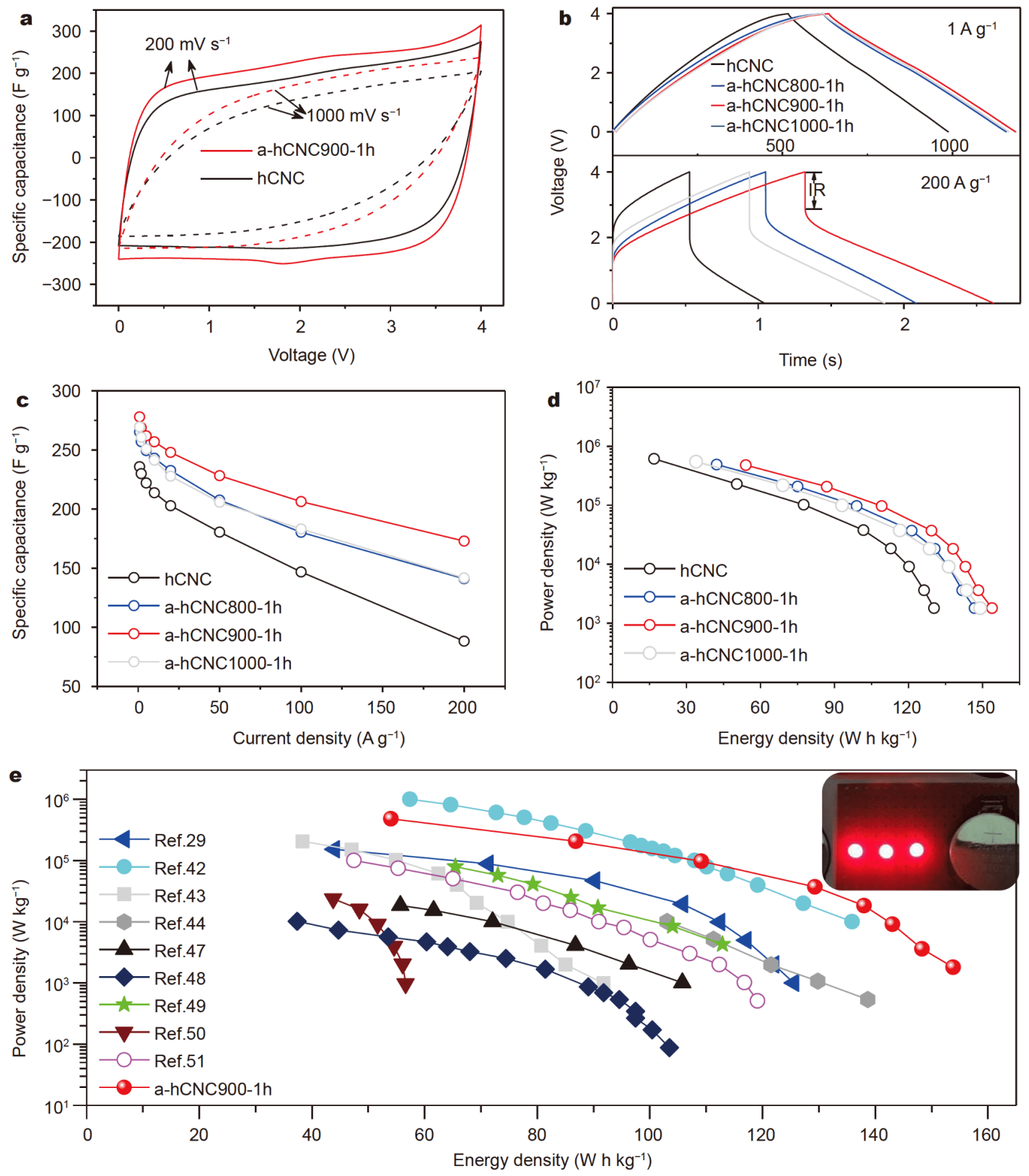

Figure 3 Supercapacitor performance of the products in $\mathrm{EMIMBF}_{4}$. (a) CV curves of the a-hCNC900-1h-SC and hCNC-SC at the scan rates of 200 and $1000 \mathrm{mV} \mathrm{s}^{-1}$, respectively. (b) GCD curves at current densities of 1 and $200 \mathrm{Ag}^{-1}$, respectively. (c) Specific capacitances at different chargedischarge current densities. (d) Ragone plots of the average-power density versus energy density of supercapacitors. (e) Ragone plots of average-power density versus energy density of the supercapacitor based on a-hCNC900-1h (in red). The other data are taken from the literatures for the typical highperformance carbon-based supercapacitors for comparison [29,42-44,47-51]. Inset shows three parallel LEDs lighted by an a-hCNC900-1h-SC.

limited as evaluated by Raman spectra, XPS, and FTIR spectroscopy (Fig. S11). All these results indicate the effectiveness to elevate the supercapacitive performance of hCNC in $\mathrm{EMIMBF}_{4}$ by properly enlarging the micropore size. Inset in Fig. 3e demonstrates the lighting of three parallel light-emitting diodes (LEDs) powered by an ahCNC900-1h-SC.
EIS was performed to further understand the correlation of the supercapacitive performance with the structural evolution, as shown in Fig. 4. The nearly vertical Nyquist plots in low-frequency range demonstrate the ideal capacitive behavior for all the supercapacitors, in which the a-hCNC900-1h-SC is the best (Fig. 4a) $[28,51]$. The enlarged high-frequency region presents a transition 

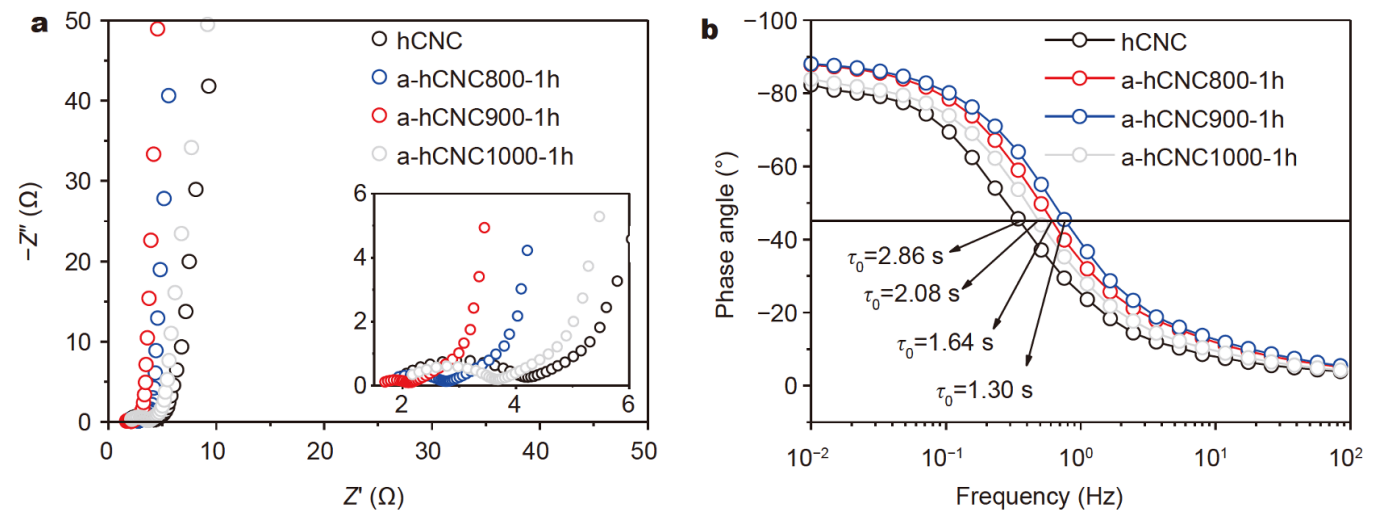

Figure 4 Frequency responses of the supercapacitors. (a) Nyquist plots. Inset magnifies the high-frequency range. (b) Bode plots of phase angle versus frequency.

from the vertical curve to the $45^{\circ}$ Warburg region followed by a semicircle (inset in Fig. 4a). By extrapolating the vertical portion of the plot to the real axis, the $R_{\mathrm{ESR}}$ values of $5.49,4.04,3.22$ and $4.81 \Omega$ were obtained for the hCNC-SC, a-hCNC800-1h-SC, a-hCNC900-1h-SC and ahCNC1000-1h-SC, respectively, in agreement with the $\mathrm{CV}$ and GCD results (Fig. 3a, b and Figs S4, S5). As known, $R_{\mathrm{ESR}}$ is mainly composed of the intrinsic Ohmic resistance $\left(R_{\mathrm{s}}\right.$, the first intercept along the real axis), the interfacial charge (ion and electron) transfer resistance $\left(R_{\mathrm{ct}}\right.$, the diameter of the semicircle), and the Warburg diffusion resistance $\left(R_{\mathrm{w}}\right.$, the projected length of Warburg region on the real axis), in which $R_{\mathrm{s}}$ mainly relies on the electrode conductivity, while $R_{\mathrm{ct}}$ and $R_{\mathrm{w}}$ are associated with the charge transfer at the electrode/electrolyte interface and the ion diffusion within pores [28]. $R_{s}$ are $1.98,1.93,1.69$ and $2.17 \Omega$ for the hCNC-SC, ahCNC800-1h-SC, a-hCNC900-1h-SC and a-hCNC1000lh-SC, respectively, consistent with the conductivity evolution (inset in Fig. 4a and Fig. S3d). Due to the similar wettability for all the products in $\mathrm{EMIMBF}_{4}$, the $R_{\mathrm{ct}}$ and $R_{\mathrm{w}}$ in this study should be mainly determined by the pore structure and conductivity of the products (Fig. S1) [28]. With increasing the activation temperature, $R_{\mathrm{ct}}$ and $R_{\mathrm{w}}$ decrease from 2.20 and $1.31 \Omega$ for the hCNC-SC to 0.85 and $1.26 \Omega$ for the a-hCNC800-1h-SC, and then 0.42 and $1.11 \Omega$ for the a-hCNC900-1h-SC. This change could be ascribed to the increase of micropore size and conductivity of the electrode materials, which favors the charge transfer. Further increasing to $1000^{\circ} \mathrm{C}$ leads to the rebound of $R_{\mathrm{ct}}$ and $R_{\mathrm{w}}$ to 1.50 and $1.14 \Omega$ for the ahCNC1000-1h-SC due to the decrease of conductivity. In the Bode plots, the characteristic frequencies $\left(f_{0}\right)$ at the phase angle of $-45^{\circ}$ for the hCNC-SC, a-hCNC800- $1 \mathrm{~h}$ SC, a-hCNC900-1h-SC and a-hCNC1000-1h-SC are 0.35,
$0.61,0.77$ and $0.48 \mathrm{~Hz}$, corresponding to the time constants $\left(\tau_{0}=1 / f_{0}\right)$ of $2.86,1.64,1.30$ and $2.08 \mathrm{~s}$, respectively (Fig. 4b). The decreasing $\tau_{0}$ from hCNC-SC to ahCNC800-1h-SC and then a-hCNC900-1h-SC also indicates the increasing micropore size [52]. The slight rebound of $\tau_{0}$ for a-hCNC1000-1h-SC should be ascribed to the partial collapse of the cage structure (Fig. S3). The shortest $\tau_{0}$ for the a-hCNC900-1h-SC among the four supercapacitors further demonstrates its fastest charge/ discharge rate and power output.

\section{CONCLUSIONS}

In summary, Boudouard reaction $\left(\mathrm{C}+\mathrm{CO}_{2}=2 \mathrm{CO}\right)$ was successfully adopted to properly enlarge the size of micropore channels on the shell of hCNC, which is accompanied by the simultaneous increase of the SSA, pore volume and conductivity while remaining the hierarchical framework of hCNC under optimal activation temperature. Such a modification boosts the ion transfer of the large-sized ionic liquid electrolyte of $\mathrm{EMIMBF}_{4}$ with a high operating voltage $(4 \mathrm{~V})$ and endows more charge storage space, which leads to the obvious decrease of equivalent series resistance and thus the dramatic increase of supercapacitive performance thereof. The optimized a-hCNC-900-1h exhibits a top-level capacitance of $278 \mathrm{~F} \mathrm{~g}^{-1}$ at $1 \mathrm{~A} \mathrm{~g}^{-1}$ for the carbon-based materials in ionic liquid electrolytes, along with a superior rate capability and long cycle life. The high energy density was achieved up to $153.8 \mathrm{~W} \mathrm{~h} \mathrm{~kg}^{-1}$ at the power density of $1.8 \mathrm{~kW} \mathrm{~kg}^{-1}$, and kept at $54.0 \mathrm{~W} \mathrm{~h} \mathrm{~kg}^{-1}$ even under an ultrahigh power density of $480.1 \mathrm{~kW} \mathrm{~kg}^{-1}$, which locates at the top-level for carbon-based electrode materials and suggests the great potential applications. This study clearly demonstrates the strong correlation of the supercapacitive performance with the micropore size, which 
provides an effective way to explore advanced electrode materials.

\section{Received 10 November 2020; accepted 8 January 2021; published online 1 April 2021}

1 Miller JR, Burke A. Electrochemical capacitors: challenges and opportunities for real-world applications. Electrochem Soc Interface, 2008, 17: 53-57

2 Simon P, Gogotsi Y, Dunn B. Where do batteries end and supercapacitors begin? Science, 2014, 343: 1210-1211

3 Chen X, Paul R, Dai L. Carbon-based supercapacitors for efficient energy storage. Natl Sci Rev, 2017, 4: 453-489

4 Wu Q, Yang L, Wang X, et al. From carbon-based nanotubes to nanocages for advanced energy conversion and storage. Acc Chem Res, 2017, 50: 435-444

5 Yang X, Cheng C, Wang Y, et al. Liquid-mediated dense integration of graphene materials for compact capacitive energy storage. Science, 2013, 341: 534-537

6 Conway BE. Electrochemical Supercapacitors: Scientific Fundamentals and Technological Applications. New York: Kluwer Academic/Plenum Publishers, 1999

7 Hall PJ, Mirzaeian M, Fletcher SI, et al. Energy storage in electrochemical capacitors: designing functional materials to improve performance. Energy Environ Sci, 2010, 3: 1238-1251

8 Yan J, Wang Q, Wei $\mathrm{T}$, et al. Recent advances in design and fabrication of electrochemical supercapacitors with high energy densities. Adv Energy Mater, 2014, 4: 1300816

9 Béguin F, Presser V, Balducci A, et al. Supercapacitors: Carbons and electrolytes for advanced supercapacitors. Adv Mater, 2014, 26: 2283

10 Wang X, Mehandzhiyski AY, Arstad B, et al. Selective charging behavior in an ionic mixture electrolyte-supercapacitor system for higher energy and power. J Am Chem Soc, 2017, 139: 18681-18687

11 Wang DW, Li F, Liu M, et al. 3D aperiodic hierarchical porous graphitic carbon material for high-rate electrochemical capacitive energy storage. Angew Chem Int Ed, 2008, 47: 373-376

12 Luo J, Jang HD, Huang J. Effect of sheet morphology on the scalability of graphene-based ultracapacitors. ACS Nano, 2013, 7: 1464-1471

13 Zheng X, Luo J, Lv W, et al. Two-dimensional porous carbon: synthesis and ion-transport properties. Adv Mater, 2015, 27: 53885395

14 Wang Q, Yan J, Fan Z. Carbon materials for high volumetric performance supercapacitors: design, progress, challenges and opportunities. Energy Environ Sci, 2016, 9: 729-762

15 Shao $\mathrm{H}, \mathrm{Wu}$ YC, Lin Z, et al. Nanoporous carbon for electrochemical capacitive energy storage. Chem Soc Rev, 2020, 49: 30053039

16 Ma H, Geng H, Yao B, et al. Highly ordered graphene solid: an efficient platform for capacitive sodium-ion storage with ultrahigh volumetric capacity and superior rate capability. ACS Nano, 2019, 13: $9161-9170$

17 Zhai Y, Dou Y, Zhao D, et al. Carbon materials for chemical capacitive energy storage. Adv Mater, 2011, 23: 4828-4850

18 Sevilla M, Mokaya R. Energy storage applications of activated carbons: supercapacitors and hydrogen storage. Energy Environ Sci, 2014, 7: 1250-1280

19 El-Kady MF, Shao Y, Kaner RB. Graphene for batteries, supercapacitors and beyond. Nat Rev Mater, 2016, 1: 16033
20 Salunkhe RR, Kaneti YV, Kim J, et al. Nanoarchitectures for metal-organic framework-derived nanoporous carbons toward supercapacitor applications. Acc Chem Res, 2016, 49: 2796-2806

21 Salunkhe RR, Lee YH, Chang KH, et al. Nanoarchitectured graphene-based supercapacitors for next-generation energy-storage applications. Chem Eur J, 2014, 20: 13838-13852

22 Jiang XF, Li R, Hu M, et al. Zinc-tiered synthesis of 3D graphene for monolithic electrodes. Adv Mater, 2019, 31: 1901186

23 Wu Q, Yang L, Wang X, et al. Carbon-based nanocages: A new platform for advanced energy storage and conversion. Adv Mater, 2019, 1904177

24 Wu Q, Yang L, Wang X, et al. Mesostructured carbon-based nanocages: an advanced platform for energy chemistry. Sci China Chem, 2020, 63: 665-681

25 Xie K, Qin X, Wang X, et al. Carbon nanocages as supercapacitor electrode materials. Adv Mater, 2012, 24: 347-352

26 Zhao J, Lai H, Lyu Z, et al. Hydrophilic hierarchical nitrogendoped carbon nanocages for ultrahigh supercapacitive performance. Adv Mater, 2015, 27: 3541-3545

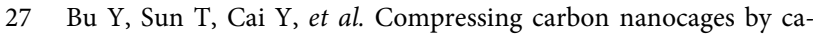
pillarity for optimizing porous structures toward ultrahighvolumetric-performance supercapacitors. Adv Mater, 2017, 29: 1700470

28 Zhao J, Jiang Y, Fan $\mathrm{H}$, et al. Porous 3D few-layer graphene-like carbon for ultrahigh-power supercapacitors with well-defined structure-performance relationship. Adv Mater, 2017, 29: 1604569

29 Lyu Z, Xu D, Yang L, et al. Hierarchical carbon nanocages confining high-loading sulfur for high-rate lithium-sulfur batteries. Nano Energy, 2015, 12: 657-665

30 Sing KSW. Reporting physisorption data for gas/solid systems with special reference to the determination of surface area and porosity (Recommendations 1984). Pure Appl Chem, 1985, 57: 603-619

31 Cychosz KA, Guillet-Nicolas R, García-Martínez J, et al. Recent advances in the textural characterization of hierarchically structured nanoporous materials. Chem Soc Rev, 2017, 46: 389-414

32 Osswald S, Portet C, Gogotsi Y, et al. Porosity control in nanoporous carbide-derived carbon by oxidation in air and carbon dioxide. J Solid State Chem, 2009, 182: 1733-1741

33 Shen L, Sun T, Zhuo O, et al. Alcohol-tolerant platinum electrocatalyst for oxygen reduction by encapsulating platinum nanoparticles inside nitrogen-doped carbon nanocages. ACS Appl Mater Interfaces, 2016, 8: 16664-16669

34 Wang X, Li X, Zhang L, et al. N-doping of graphene through electrothermal reactions with ammonia. Science, 2009, 324: 768771

35 Tsang SC, Harris PJF, Green MLH. Thinning and opening of carbon nanotubes by oxidation using carbon dioxide. Nature, 1993, 362: 520-522

36 Babu DJ, Yadav S, Heinlein T, et al. Carbon dioxide plasma as a versatile medium for purification and functionalization of vertically aligned carbon nanotubes. J Phys Chem C, 2014, 118: 1202812034

37 Zhang LL, Zhao X, Stoller MD, et al. Highly conductive and porous activated reduced graphene oxide films for high-power supercapacitors. Nano Lett, 2012, 12: 1806-1812

38 Zhang J, Jia K, Lin L, et al. Large-area synthesis of superclean graphene via selective etching of amorphous carbon with carbon dioxide. Angew Chem Int Ed, 2019, 58: 14446-14451

39 Han X, Funk MR, Shen F, et al. Scalable holey graphene synthesis and dense electrode fabrication toward high-performance ultra- 
capacitors. ACS Nano, 2014, 8: 8255-8265

$40 \mathrm{Xu}$ J, Tan Z, Zeng W, et al. A hierarchical carbon derived from sponge-templated activation of graphene oxide for high-performance supercapacitor electrodes. Adv Mater, 2016, 28: 5222-5228

41 Li C, Zhang X, Wang $\mathrm{K}$, et al. Scalable self-propagating hightemperature synthesis of graphene for supercapacitors with superior power density and cyclic stability. Adv Mater, 2017, 29: 1604690

42 Li J, Wang N, Tian J, et al. Cross-coupled macro-mesoporous carbon network toward record high energy-power density supercapacitor at 4 V. Adv Funct Mater, 2018, 28: 1806153

43 Yao L, Wu Q, Zhang $\mathrm{P}$, et al. Scalable 2D hierarchical porous carbon nanosheets for flexible supercapacitors with ultrahigh energy density. Adv Mater, 2018, 30: 1706054

44 Bo Z, Zhu W, Ma W, et al. Vertically oriented graphene bridging active-layer/current-collector interface for ultrahigh rate supercapacitors. Adv Mater, 2013, 25: 5799-5806

45 Pachfule P, Shinde D, Majumder M, et al. Fabrication of carbon nanorods and graphene nanoribbons from a metal-organic framework. Nat Chem, 2016, 8: 718-724

46 Cui C, Qian W, Yu Y, et al. Highly electroconductive mesoporous graphene nanofibers and their capacitance performance at $4 \mathrm{~V}$. J Am Chem Soc, 2014, 136: 2256-2259

47 Hou J, Cao C, Idrees F, et al. Hierarchical porous nitrogen-doped carbon nanosheets derived from silk for ultrahigh-capacity battery anodes and supercapacitors. ACS Nano, 2015, 9: 2556-2564

48 Niu J, Shao R, Liang J, et al. Biomass-derived mesopore-dominant porous carbons with large specific surface area and high defect density as high performance electrode materials for Li-ion batteries and supercapacitors. Nano Energy, 2017, 36: 322-330

49 Nomura K, Nishihara H, Kobayashi N, et al. $4.4 \mathrm{~V}$ supercapacitors based on super-stable mesoporous carbon sheet made of edge-free graphene walls. Energy Environ Sci, 2019, 12: 1542-1549

50 Shang T, Xu Y, Li P, et al. A bio-derived sheet-like porous carbon with thin-layer pore walls for ultrahigh-power supercapacitors. Nano Energy, 2020, 70: 104531

51 Shao Y, El-Kady MF, Lin CW, et al. 3D freeze-casting of cellular graphene films for ultrahigh-power-density supercapacitors. Adv Mater, 2016, 28: 6719-6726

52 Chmiola J, Yushin G, Gogotsi Y, et al. Anomalous increase in carbon capacitance at pore sizes less than 1 nanometer. Science, 2006, 313: 1760-1763

Acknowledgements This work was jointly supported by the National Key Research and Development Program of China (2017YFA0206500 and 2018YFA0209103), the National Natural Science Foundation of China (21832003, 21773111, 21573107 and 21971061), and the Fundamental Research Funds for the Central Universities (020514380126).

Author contributions $\mathrm{Hu}$ Z, Wang X, Ma Y and Zhao J conceived the concept and designed the experiments. Zhao J carried out the experiments. Fan $\mathrm{H}$ and Li G assisted with experiments and data preparation. Yang $\mathrm{L}$ performed the theoretical simulation. $\mathrm{Hu} \mathrm{Z}, \mathrm{Wu} \mathrm{Q}$ and Zhao J wrote the manuscript. All authors analyzed and discussed the data and commented on the manuscript.

Conflict of interest The authors declare no conflict of interest.

Supplementary information online version of the paper.

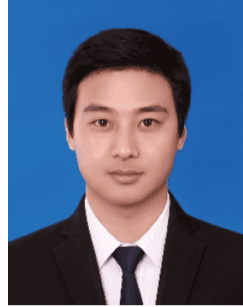

Jin Zhao is currently a professor at Nanjing University of Posts and Telecommunications. He received his ME degree from Nanjing University of Posts and Telecommunications in 2012 and PhD degree from Nanjing University in 2016. After that, he worked as a research fellow at the National University of Singapore and Nanyang Technological University. His research focuses on the design and preparation of advanced electrode materials for energy storage and conversion.

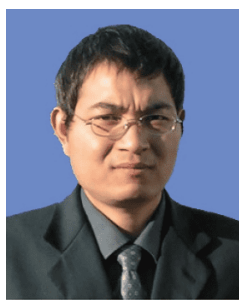

Xizhang Wang received his $\mathrm{PhD}$ in chemistry from Nanjing University in 2001. He was appointed an associate professor of Nanjing University in 2003 and full professor in 2011. He had been a Japan Society for Promotion of Science Fellow in Tokyo University (2003-2005). His scientific interests mainly focus on nanomaterial chemistry, sustainable energy, and heterogeneous catalysis.

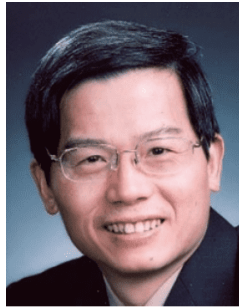

Zheng Hu received his BSc (1985) and $\mathrm{PhD}$ (1991) degrees in physics from Nanjing University. After two-year's postdoctoral research in the Department of Chemistry, he became an associate professor in 1993, and subsequently acquired the professor position in 1999, and Cheung Kong Scholar professor in 2007. He was awarded the NSFC fund for outstanding young scientists of China (2005). He is engaged in the research field of physical chemistry and materials chemistry addressing the growth mechanism, materials design and energy applications of a range of nano-/mesostructured materials, especially the carbon nanocages, group III nitrides and transition metal oxides.

\section{增大分级结构碳纳米笼的离子传输微孔通道获得 超高的能量密度和功率密度}

赵进 ${ }^{1,2}$, 范豪 ${ }^{1}$, 李国昌 ${ }^{1}$, 吴强 ${ }^{1}$, 杨立军 ${ }^{1}$, 马延文 ${ }^{2}$, 王喜章 ${ }^{1 *}$, 胡征 ${ }^{1 *}$

摘要 提升超级电容器能量密度而不牺牲其高功率密度是能源存 储领域的不解追求. 离子液体电解液具有大的工作电压窗口，以其 为电解液可提升超级电容器能量密度. 但离子液体离子尺寸大、 离子电导率低且粘度高, 通常会导致超级电容器功率密度的减小. 本文主要通过增大分级结构碳纳米笼( $\mathrm{hCNC}$ )电极材料的离子传输 微孔通道, 实现了在 $\mathrm{EMIMBF}_{4}$ 离子液体电解液中超级电容器能量 密度和功率密度的协同提升. 通过Boudouard反应, 在保持 $\mathrm{hCNC}$ 独特分级结构的前提下调节了贯穿碳纳米笼壁的微孔尺寸，同时， 在优化的活化温度下 $\mathrm{hCNC}$ 的比表面积、孔体积和导电性均得以 提升. 这种独特的调节促进了大尺寸离子的传输, 有效降低了等效 串联电阻, 从而显著提升了超级电容器的性能. 优化的样品在功率 密度为 $1.8 \mathrm{~kW} \mathrm{~kg}^{-1}$ 时的能量密度高达 $153.8 \mathrm{~W} \mathrm{~h} \mathrm{~kg}^{-1}$, 在超高功率 密度 $480.1 \mathrm{~kW} \mathrm{~kg}^{-1}$ 时能量密度仍能保持 $54.0 \mathrm{~W} \mathrm{~h} \mathrm{~kg}^{-1}$. 本研究展示 了一种通过精细调控材料微孔及相关性质开发先进电极材料的有 效方法. 